\title{
Modelo de valoración patrimonial de un elemento singular: la cúpula de la escuela técnica superior de ingenieros industriales
}

\section{Patrimonial assesment model of a singular element: the dome of the Higher Technical School of Industrial Engineers}

M. L. Martínez $^{(*)}$, A. Carretero ${ }^{(*)}$, J. D. Sanz $^{(*)}$, P. Álvarez ${ }^{(*)}$

RESUMEN

El presente trabajo persigue establecer si la cúpula del edificio de la Escuela Técnica Superior de Ingenieros Industriales de Madrid (ETSII) tiene valor como elemento representativo del patrimonio arquitectónico e industrial con el objetivo en su caso de promover su protección con la intención de afrontar su recuperación y garantizar su adecuada conservación. El estado actual de esta cúpula es claramente mejorable, sobre todo en su interior. Por este motivo, resulta indispensable garantizar un sistema de rehabilitación para este elemento, que constituye el símbolo de esta Escuela y una importante manifestación de la arquitectura de exposiciones de finales del siglo XIX.

En este trabajo se han analizado diversas opciones que pueden justificar su reconocimiento y la posterior inclusión en un programa de protección que reconozca el valor patrimonial de esta construcción, y decidiendo finalmente que la alternativa más viable es el Plan Nacional de Patrimonio Industrial (1).

Palabras clave: Patrimonio, cúpula, BIM, protección.

\section{ABSTRACT}

The present paper aims to establish if the dome of the building of the Higher Technical School of Industrial Engineers of Madrid (ETSII) has value as a representative element of the architectural and industrial heritage with the objective, if necessary, to promote its protection with the intention of facing its recovery and ensure its conservation.

The current state of this dome is clearly improveable, especially inside. For this reason, it is essential to guarantee a rehabilitation system for this element, which is the symbol of this College and an important manifestation of the architecture of the late XIX century.

In this work, we have analyzed several options that can justify its recognition and the subsequent inclusion in a protection program that recognizes the equity value of this construction, and finally deciding that the most viable alternative is the National Industrial Heritage Plan (1).

Keywords: Heritage, dome, BIM, protection.

(*) Universidad Politécnica de Madrid.

Persona de contacto/Corresponding author: luisa.mtzmuneta@upm.es (M. L. Martínez)

ORCID: http://orcid.org/oooo-0oo3-3725-7400 (M. L. Martínez); http://orcid.org/oooo-ooo1-5203-2782 (A. Carretero); http://orcid.org/oooo-0oo2-8039-8527 (J. D. Sanz); http://orcid.org/oooo-0002-4442-1777 (P. Álvarez)

Cómo citar este artículo/Citation: M. L. Martínez; A. Carretero; J. D. Sanz; P. Álvarez. (2018). Modelo de valoración patrimonial de un elemento singular: la cúpula de la escuela técnica superior de ingenieros industriales. Informes de la Construcción, 70(550): e254. https://doi.org/10.3989/id.58049

Copyright: (C) 2018 CSIC. Este es un artículo de acceso abierto distribuido bajo los términos de la licencia de uso y distribución Creative Commons Reconocimiento 4.o Internacional (CC BY 4.0). 


\section{ORIGEN DEL EDIFICIO}

El edificio se encuentra ubicado en el número 2 de la calle José Gutiérrez Abascal de Madrid, y alberga las sedes de la Escuela Técnica Superior de Ingenieros Industriales y del Museo Nacional de Ciencias Naturales (Figura 1).

En sus orígenes, había alojado el Palacio Nacional de la Industria y las Artes, una importante apuesta por la innovación en la España de finales del siglo XIX.

A partir del año 1856 durante más de un siglo se comenzaron a desarrollar las Exposiciones Nacionales de Bellas Artes, la última exhibición fue en el año 1968. Estos certámenes estaban regidos según lo dispuesto en el Real Decreto de Isabel II el 28 de diciembre de 1853 .

Según este decreto, estas exposiciones debían ser bienales, y se establecía que tendría lugar una exposición pública de obras de Bellas Artes durante el mes de mayo. Sin embargo, no llegaron a tener esta periodicidad.

Con el objetivo de construir un edificio adecuado para albergar este tipo de eventos, y en concreto de la celebración de la Exposición Nacional de la Industria y de las Artes en 1881(2), se convocó un concurso público que ganó el arquitecto Fernando de la Torriente. La propuesta consistía en un edificio de planta rectangular con dos patios interiores, un cuerpo central adelantado que formaba la entrada y un remate posterior con un ábside. Todo ello estaría coronado por una cúpula sobre tambor de ocho gajos (Figura 2). Este edificio es un claro exponente de la denominada "arquitectura del hierro" en la ciudad de Madrid.
Desde 1907, la Escuela Técnica Superior de Ingenieros Industriales comparte el edificio con el Museo de Ciencias Naturales. Desde entonces se han realizado reformas, rehabilitaciones y edificios auxiliares anexos a la construcción original. Se destaca la modificación que sufrió como consecuencia del centenario de la carrera de Ingeniería Industrial en 1950-1952, transformando el salón bajo la cúpula en salón de actos, y la reforma de la que hoy es conocida como la Sala de la Máquina, así como la incorporación de diferentes elementos ornamentales en el hall de entrada y en dicha sala. Esta reforma ocultó la estructura metálica original de esta sala bajo una serie de pilares recubiertos de mármol que se mantiene en la actualidad.

\section{CARACTERÍSTICAS DE LA CÚPULA}

El sistema estructural reconocible en la construcción de esta parte singular del edificio es el denominado sistema Polonceau (3). La base de la construcción es un octógono de 8,50 $\mathrm{m}$ de lado cuyos vértices descansan sobre una circunferencia de 22,30 m de diámetro. La superficie de la cúpula alcanza una altura de $20,80 \mathrm{~m}$. A esto hay que sumar la altura del cupulín, con una linterna de 3,10 $\mathrm{m}$ y una parte superior que añade $2 \mathrm{~m}$ más. En total, sumando las dimensiones del pináculo situado en el punto más alto para alojar el pararrayos, el conjunto asciende $28,70 \mathrm{~m}$ desde el tejado del edificio.

El acceso al exterior de la cúpula se realiza a través de la cubierta del edificio, y únicamente es posible caminar sobre una estrecha plataforma de pocos metros de longitud habilitada a tal efecto. El ascenso al cupulín no es posible actualmente, pues la escalera existente para ello fue eliminada en la última rehabilitación (2007) debido a su mal estado de conservación.

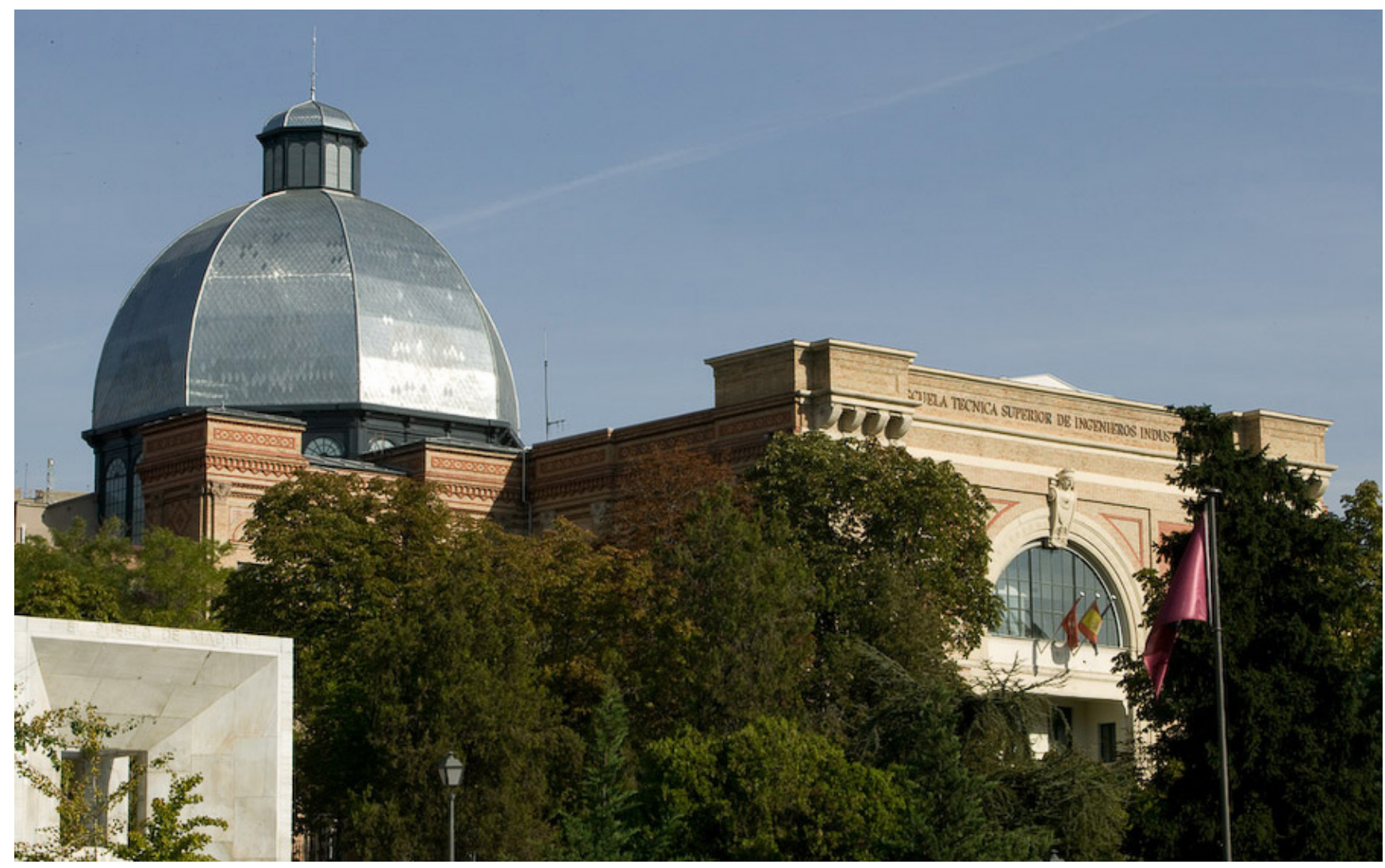

Figura 1. Vista general del edificio en la actualidad. Fuente: Archivo gráfico de la ETSII. 

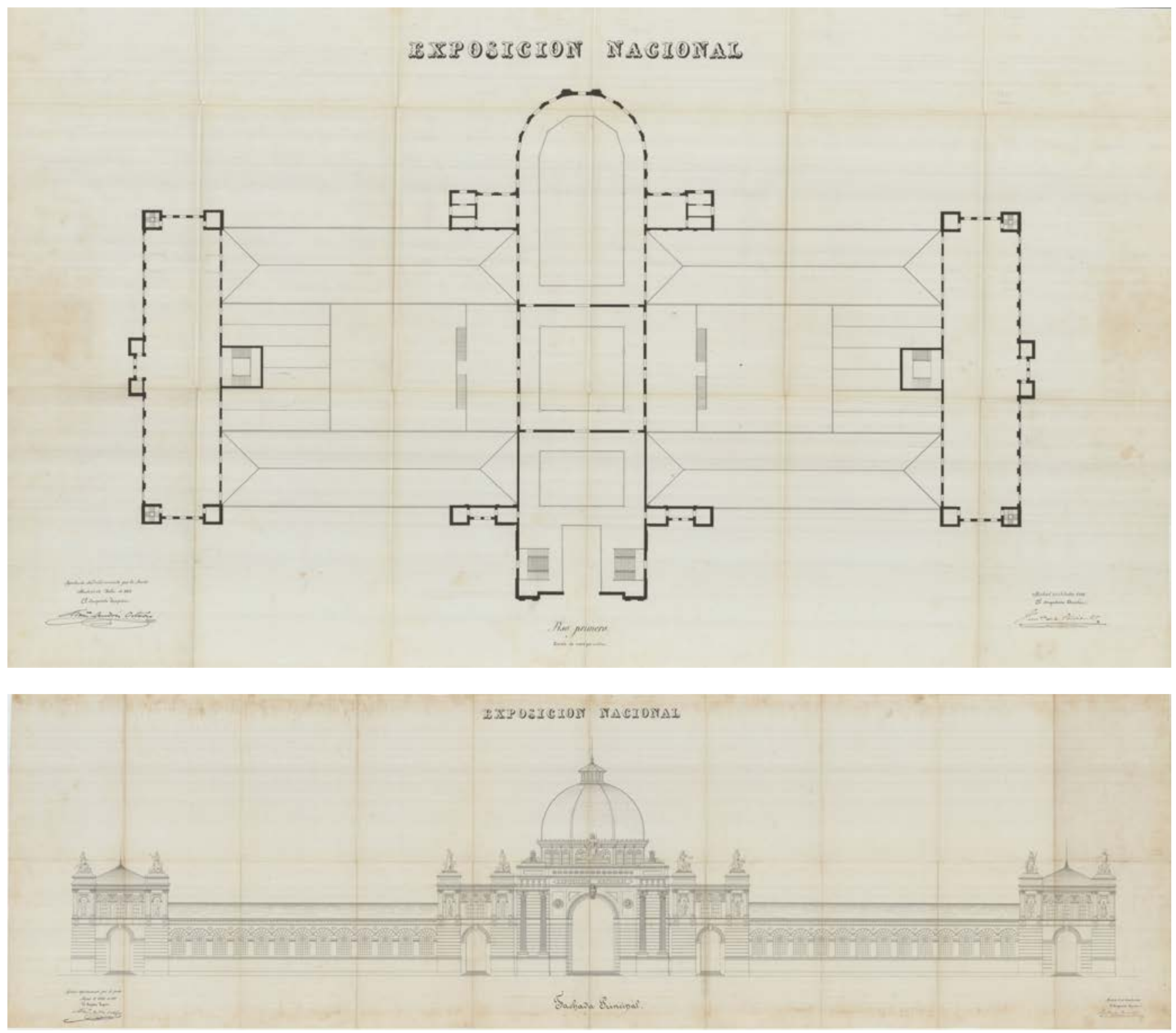

Figura 2. Planos originales del Palacio de la Industria y las Artes.

El piso inmediatamente inferior, con una profundidad de $3,25 \mathrm{~m}$ respecto a la base octogonal del tambor, se encuentra sustentado por 32 arcos de hierro decorados con forjados de motivos vegetales con referencia neoclásica que aportan sensación de armonía y estilizan el arco. Existen tres tipos de arcos según sus dimensiones, y en todos ellos se aprecian estos bajorrelieves subordinados al marco arquitectónico, esto es, el adorno se adapta a la arquitectura. En la actualidad es necesario, además, el sustento de puntales provisionales de madera debido al riesgo de desprendimientos y apenas la cuarta parte del suelo de este piso está asegurado y es accesible con garantías de seguridad (Figura 3).

El número de tejas de zinc se estima a partir de las fotografías y del modelo realizados, y resulta ser de 9.080 para la superficie de la cúpula y de 912 para la del cupulín.

Las dimensiones de las tejas son de $40,5 \times 21,8 \mathrm{~cm}$ en el caso del cupulín y de $61 \times 30,4 \mathrm{~cm}$ para las tejas de la cúpula.

Se emplearon 59.424 roblones distribuidos de la siguiente forma:
- Piso inferior: 19.568

- Ventanas tambor exterior: 12.888

- Embellecedores y canalón: 1.936

- Ventanas linterna exterior: 2.864

- Pasarela del cupulín: 1.008

- Estructura metálica cúpula: 10.432

- Estructura metálica del cupulín: 736

- Ventanas linterna interior: 3.056

Algunos de estos roblones están actualmente sustituidos por pernos.

Es interesante también la presencia en su interior de un lucernario en forma de pirámide de base cuadrada, con un lado de casi 20 metros y una altura máxima de 4 metros en su cúspide realizado en una intervención en el año 1979. La estructura que lo soporta, formada por vigas de acero y de madera, sustenta las láminas de cristal centrales y las chapas de zinc que rodean a ésta.

La cúpula comprende un volumen de 7.560 m3 y aproximadamente 400 toneladas de peso. 

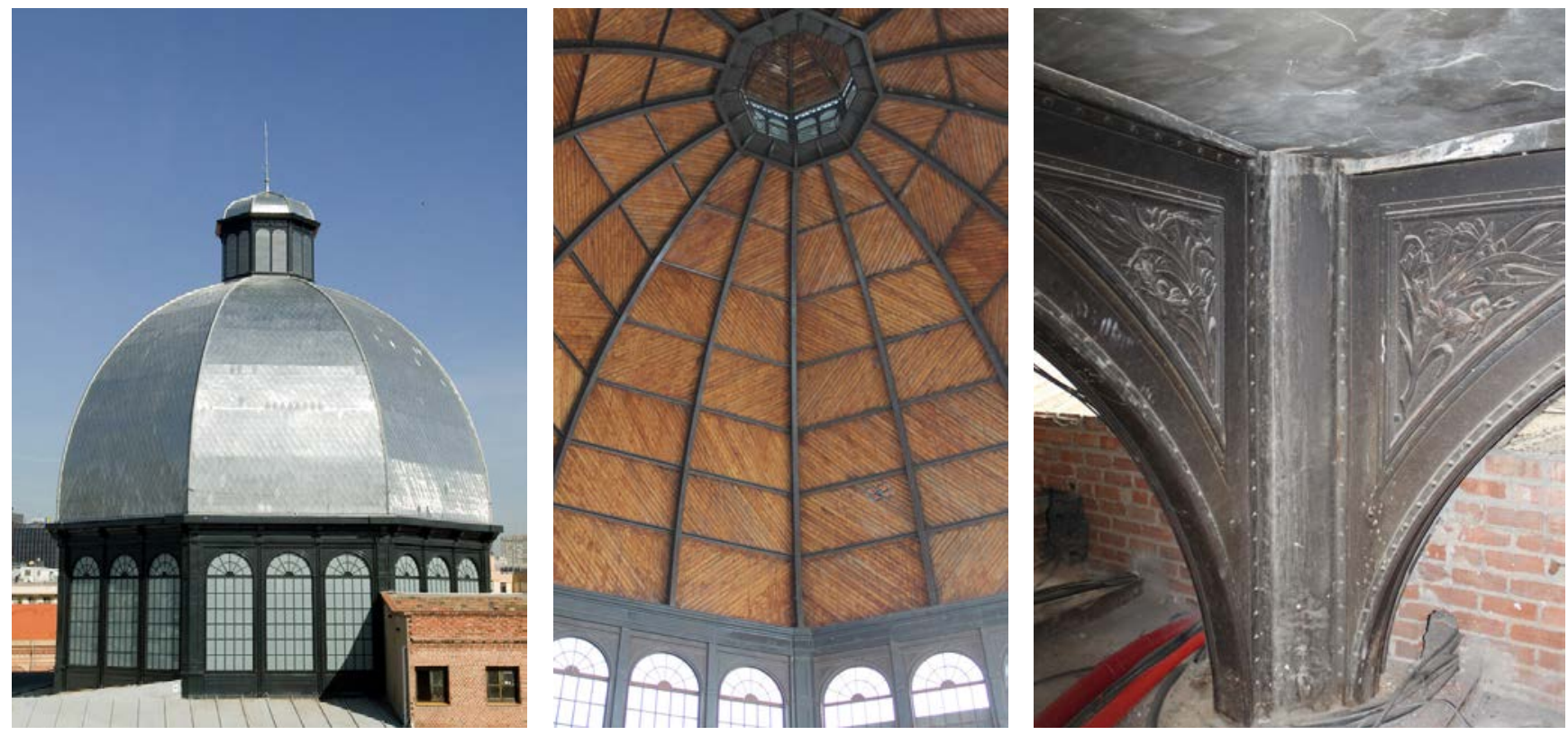

Figura 3. Exterior e interior de la cúpula de la ETSII. Fuente: autores.

\section{ACTUACIONES SOBRE EL EDIFICIO}

El edificio ha sufrido diversas modificaciones desde su creación, principalmente en la distribución interna, con el fin de adaptarlo a las diferentes funciones que ha tenido a lo largo de su historia. La documentación tanto escrita como gráfica de los mismos ha sido complicada de obtener, a veces incluso inexistente e incluso, en ocasiones, contradictoria.

En el año 1896, el arquitecto Pascual Herráiz y Silo realizó la primera gran remodelación de los espacios interiores. Estas modificaciones de menor importancia se vinieron sucediendo una función de los nuevos usos y de los ocupantes del edificio (4).

Bajo la dirección de D. Manuel Soto, Director de la Escuela Técnica Superior de Ingenieros Industriales desde 1940 a 1963, las reformas fueron constantes, aunque la documentación durante sus primeros años es prácticamente inexistente. De su mandato resultaron las zonas conocidas como "la Rotonda", el laboratorio Central de Electrotecnia y del Instituto de Investigaciones y Experiencias Cinematográficas, la recuperación del ala sur anteriormente ocupada por la Guardia Civil y la construcción de una cuarta planta donde se ubicó la biblioteca. También se crearon zonas de taller y laboratorios. Se añadió un anexo al ala sur donde se albergó la capilla y el salón de actos en dos niveles, añadiendo posteriormente otras dos plantas más para aulas de dibujo.

Es especialmente interesante para este estudio la posibilidad real que existió de desaparición de la cúpula, emblema actual de la ETSII. Este hecho queda recogido en un Plan Director efectuado por la propia Escuela. Según este documento, en la memoria del proyecto redactada por el arquitecto Manuel Blanc (5) se exponía:

"La cúpula que existe en la vertical del Salón de Actos, que en otro tiempo, al construirse este edificio para exposiciones, era vista desde el interior, hoy no tiene sentido alguno, por la transformación operada en la estructura.
En la actualidad, esta cúpula se encuentra en muy malas condiciones de conservación (entre otras razones porque el acceso a ella es muy difícil y peligroso), siendo un continuo foco de humedades irreparables. No obstante, a fin de solucionar este problema se corrigieron no hace mucho tiempo las bajadas pluviales, se enmasillaron cristales y se repusieron los rotos. A pesar de todo, las humedades persisten, debido a que la cubierta de hojas de zinc ha perdido efectividad. La reparación de toda ella sería extraordinariamente costosa, estando siempre sometida al peligro de su difícil conservación.

Otras razones abundan también sobre la necesidad de su demolición.

Iniciadas unas obras de adaptación al nuevo plan de estudios de 1958, se resolvió con criterio lógico, la composición de fachadas de acuerdo a una estética más actual. El pretender mantener unas formas caducas en el aspecto de un edificio que alberga una organización y unas necesidades actuales hubiera sido falso y costoso. Iniciados pues, en esta renovación el criterio que se ha seguido en nuevas ampliaciones, ha estado presidido por la misma idea de actualidad. A la hora de estar afectado por la reforma la totalidad del edificio, su unidad requiere el mismo criterio de ordenación. Su fachada principal debe estar sujeta a esta renovación y así se prevé en este proyecto. La conservación de la cúpula sería entonces un absurdo volumétrico y de sentido.

Ninguna razón puede abogar por la conservación de un elemento al que ya se sentenció a desaparecer en el momento en que, por las reformas anteriores de construcción de entreplantas se suprimió su función."

Años más tarde tuvieron lugar nuevas remodelaciones en el año 1979, por parte de la arquitecta Amparo Berlinches Acín y se actuó sobre la fachada y la cúpula, con el objetivo de recuperar el lucernario y las cerámicas de los famosos Juan Ruíz de Luna y Daniel Zuloaga. Se incorporó en el interior una pirámide de cuatros caras de material plástico para reconducir las posibles goteras y evitar inundaciones por la cúpula. 


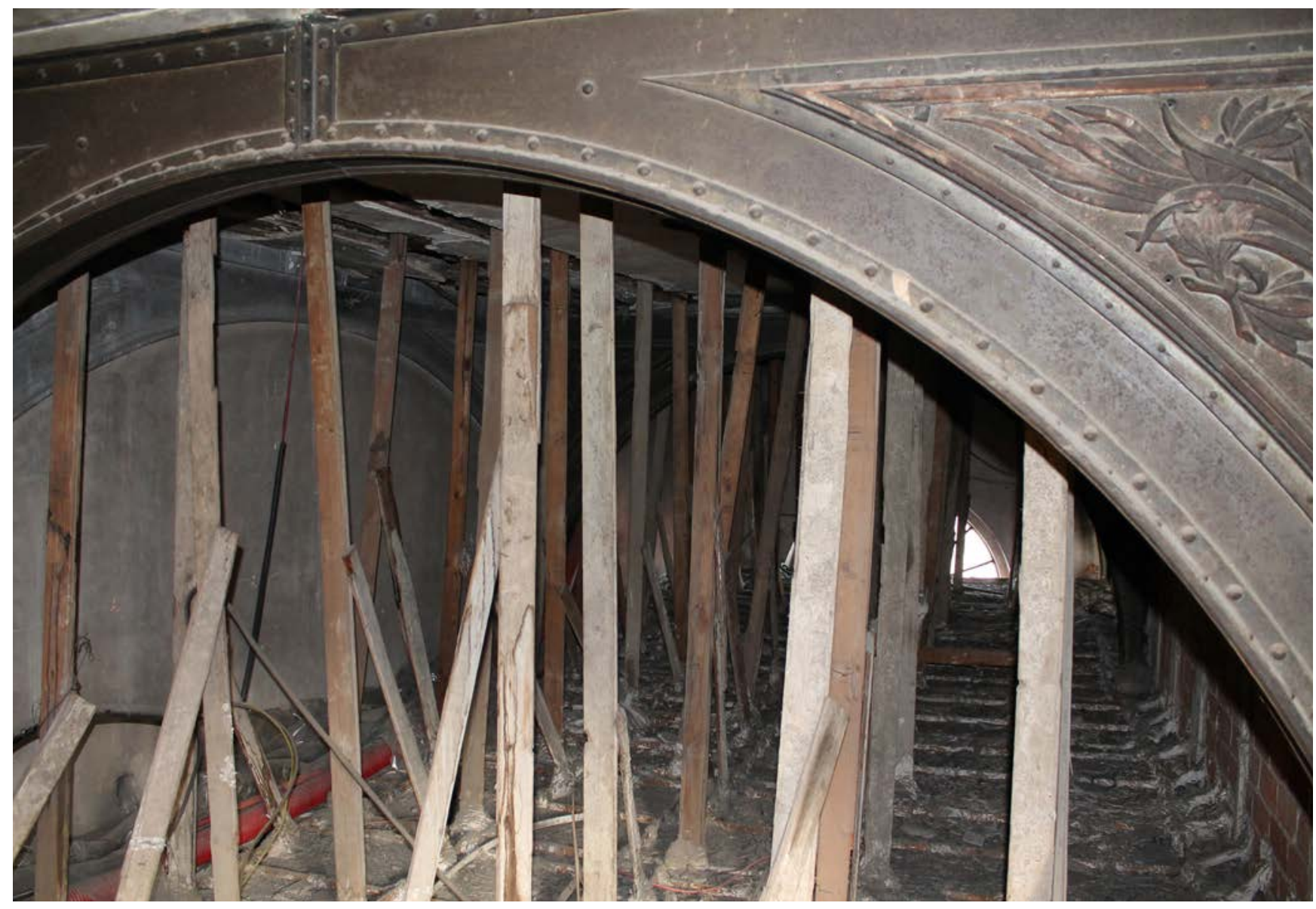

Figura 4. Estado actual del interior de la cúpula. Fuente: autores.

Las siguientes intervenciones fueron más respetuosas y fueron acompañadas de un estudio histórico del edificio (6).

En 1999 se abrió una tercera planta en el edificio central, ocupando un espacio perdido y empleado como desván y recuperando las columnas originales de hierro forjado que estaban ocultas.

Más recientemente, en 2007, con motivo del desprendimiento de tejas y canalones producido por los fuertes vientos de febrero de ese mismo año, fue necesario un nuevo proyecto de restauración. Este plan fue encabezado por los arquitectos Ana María Montiel y José María del Monte (6).

Las obras efectuadas incluyeron tareas como la reposición total de la cobertura de zinc, la supervisión y reparación de la madera base de la misma y la estructura y cerramientos de acero, tanto de la cúpula, como del tambor sobre el que se sustenta (7). Según el Plan Director del año 2009 (9) y como después confirmaría el entonces Director de la Escuela en un artículo en El País (10), esta reforma tuvo un coste de $765.067 €$.

\section{ESTUDIO DEL INTERÉS PATRIMONIAL}

Se ha realizado un estudio del interés patrimonial sin perder de vista el objetivo general del proyecto: la restauración y la posterior conservación de la cúpula de la Escuela Técnica Superior de Ingenieros Industriales de Madrid, con la intención de poder aprovechar este espacio en el futuro (Figura 4).
Se han planteado diversas opciones para intentar alcanzar este objetivo y se ha analizado que la mejor manera de llevarlo a cabo es conseguir incluir la cúpula en un catálogo de bienes protegidos. De nuevo aparecen distintas alternativas, pues existen multitud de grados de protección y éstas se revisan para decidir cuál es la más viable para el caso de estudio. Son de especial relevancia por su actividad de salvaguarda y protección de los bienes, de forma que sean preservados para servir de objeto de estudio a las generaciones futuras.

\section{NIVELES DE PROTECCIÓN}

Con el fin de encuadrar a la cúpula de la ETSII dentro de un catálogo de bienes protegidos se han revisado las siguientes posibilidades de proteccion existentes:

- PATRIMONIO DE LA HUMANIDAD (UNESCO): No es posible esta protección para la cúpula de la ETSII: primero tiene que ser reconocido como Bien de Patrimonio Local o Nacional. Es demasiado ambicioso: exige ser un ejemplo sobresaliente, representar una obra maestra del genio creativo humano, ser testimonio excepcional... (11).

- PATRIMONIO ARQUITECTÓNICO (ICOMOS: Consejo Internacional de Monumentos y Sitios): Aplicado al ámbito internacional, exige criterios muy ambiciosos (12) (13).

- PATRIMONIO HISTÓRICO NACIONAL (CCAA): Algunas Comunidades Autónomas, como Andalucía, Aragón, Canarias o Asturias, destacan por el amplio desarrollo de una legislación propia en relación con este asunto. Sin 

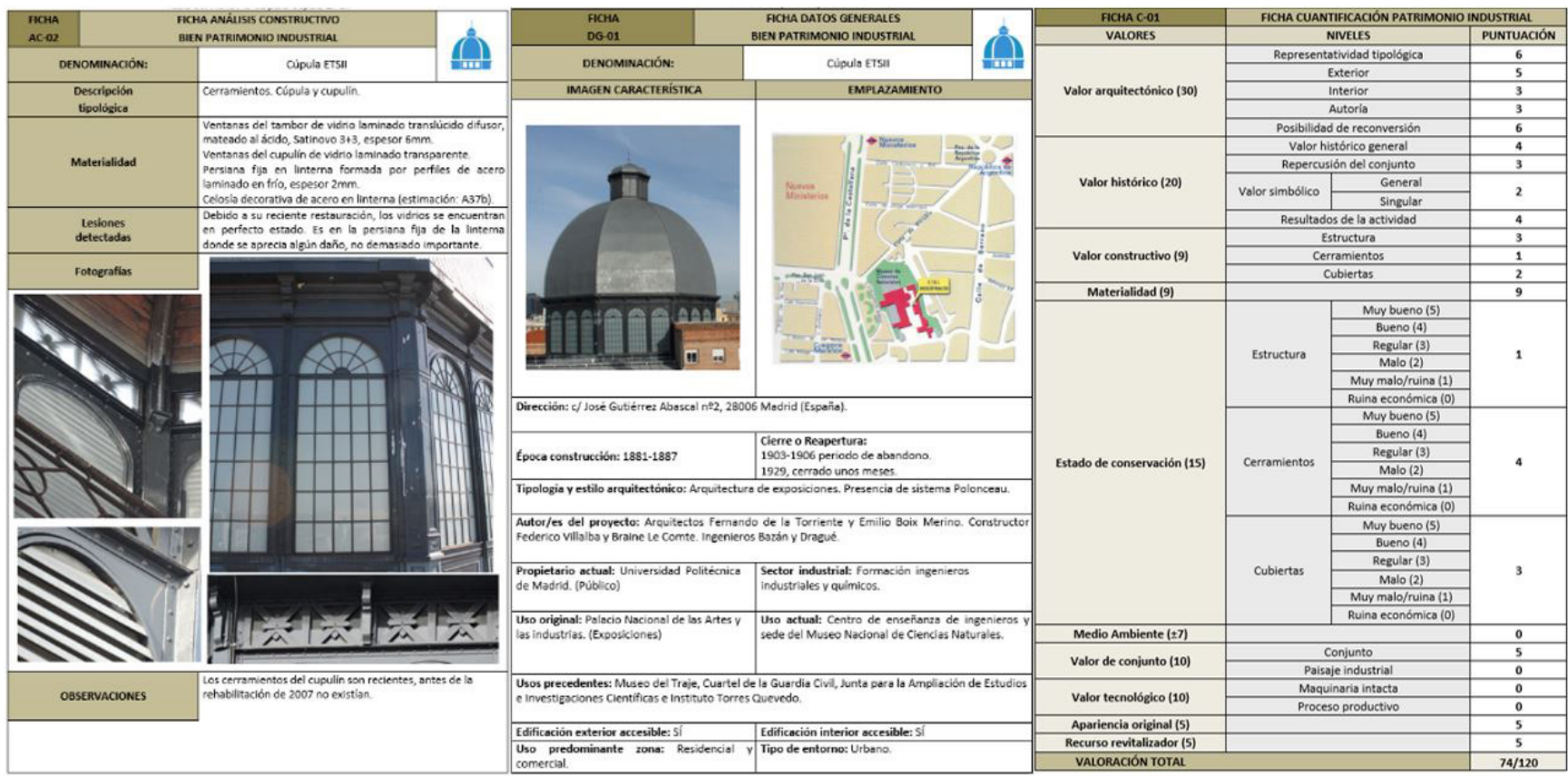

Figura 5. Fichas resumen del estudio para Patrimonio Industrial.

embargo, en el caso de la Comunidad de Madrid, el Consejo Regional de Patrimonio Histórico aplica la ley estatal (14). La documentación oficial plantea requisitos poco detallados.

- PATRIMONIO INDUSTRIAL (IPCE: Instituto Patrimonio Cultural de España). Ámbito nacional. Atendiendo a estas características, resulta evidente la relación de la cúpula estudiada con el Patrimonio Industrial español: desde sus orígenes, como uno de los elementos arquitectónicos principales del Palacio Nacional de la Industria y las Artes; hasta la actualidad, como sede de la Escuela Técnica Superior de Ingenieros Industriales de Madrid, pasando por una evolución siempre unida al sector industrial de España (1).

Un punto clave del proyecto es, por tanto, la investigación necesaria para aplicar el Plan Nacional de Patrimonio Industrial del año 2011 al caso de estudio.

Según queda recogido en dicho Plan, tras la admisión del bien en cuestión en el Catálogo de Bienes Protegidos, las acciones siguientes servirían para asegurar la recuperación de la cúpula y su seguimiento posterior, asignando una partida presupuestaria a tal efecto.

Sin embargo, el proceso de valoración de los aspectos de interés del Plan de Patrimonio Industrial no es todo lo objetivo y medible que cabría esperar. Para subsanarlo, tras encontrar precedentes de metodologías de cuantificación basadas en los puntos del Plan (15), se ha propuesto un sistema de valoración más detallado en los que se contemplan diez criterios de forma objetiva. Uno de los antecedentes principales de todos los tenidos en cuenta es la Tesis Doctoral de la arquitecta Diana Sánchez Mustieles (16), que ya planteaba un modelo compatible con el Plan de Patrimonio Industrial enfocado al estudio de fábricas.

Los criterios analizados en el presente trabajo son más generales y aplicables a cualquier construcción. Los diez criterios aplicables se describen a continuación:
- Valor arquitectónico: Tiene en cuenta niveles como la representatividad tipológica, el exterior, el interior, la autoría o la posibilidad de reconversión.

- Valor histórico: Analiza la relevancia histórica del conjunto.

- Valor constructivo: Según la estructura, cerramientos, fachadas y cubiertas.

- Materialidad: Dificultad o innovación en su uso.

- Estado de conservación: Desde muy bueno hasta ruina o ruina económica.

- Medioambiente: Especialmente importante, se analizan efectos negativos y positivos.

- Valor de conjunto: Estudia el nivel de conservación en el entorno.

- Valor tecnológico: Si conserva maquinaria original, muestras del proceso...

- Apariencia original: Si se conserva el aspecto original del edificio.

- Valor como recurso revitalizador: para el entorno del conjunto estudiado.

Además de todo esto, se han generado unas fichas a modo de resumen que recogen los aspectos principales: la valoración asignada, datos generales, contenido fotográfico más relevante y una muestra de la colección de planos técnicos desarrollada (Figura 5).

Cabe destacar la profunda investigación sobre el edificio: su historia y contexto, materiales y tipo arquitectónico al que pertenece, aplicaciones a las que ha sido destinado a lo largo de su vida útil ... que se ha realizado.

Esta información enriquece el trabajo, y sirve para recopilar la gran cantidad de datos y fotografías que se encontraban diseminados hasta ahora en la documentación de antiguos proyectos arquitectónicos de restauración, Proyectos de Fin de Carrera de la ETSII, documentos del Archivo General de la Administración y páginas web relacionadas con la Escuela, el Museo de Ciencias Naturales y el edificio. 
El resultado de aplicar estos criterios aparece en la tabla 1.

Tras la aplicación de la metodología de cálculo sobre la cúpula de la ETSII, se obtiene una puntuación de 74 sobre 120 puntos.

De acuerdo a los niveles de relevancia establecidos para un bien de patrimonio industrial (Tabla 2), se asigna por tanto un nivel de relevancia 2, correspondiente a los bienes de relevancia industrial que, si bien es cierto que no es el máximo nivel posible, ya que pone de manifiesto la importancia del bien para el patrimonio nacional, y la pérdida irreparable que supondría su desaparición. Este estudio servirá para apoyar la solicitud de protección suministrada por el Plan Nacional de Patrimonio Industrial y perseguir así el objetivo planteado.

Tabla 1. Resultados obtenidos de sistema de valoración propuesto.

\begin{tabular}{|c|c|c|c|}
\hline Valores & \multicolumn{2}{|c|}{ Niveles } & Puntuación \\
\hline \multirow{5}{*}{ Valor arquitectónico (30 puntos) } & \multicolumn{2}{|c|}{ Representatividad tipológica } & 6 \\
\hline & \multicolumn{2}{|l|}{ Exterior } & 5 \\
\hline & \multicolumn{2}{|l|}{ Interior } & 3 \\
\hline & \multicolumn{2}{|l|}{ Autoría } & 3 \\
\hline & \multicolumn{2}{|c|}{ Posibilidad de reconversión } & 6 \\
\hline \multirow{5}{*}{ Valor histórico (20 puntos) } & \multicolumn{2}{|c|}{ Valor histórico general } & 4 \\
\hline & \multicolumn{2}{|c|}{ Repercusión del conjunto } & 3 \\
\hline & \multirow{2}{*}{ Valor simbólico } & General & \multirow{2}{*}{2} \\
\hline & & Singular & \\
\hline & \multicolumn{2}{|c|}{ Resultados de la actividad } & 4 \\
\hline \multirow{3}{*}{ Valor constructivo (9 puntos) } & \multicolumn{2}{|l|}{ Estructura } & 3 \\
\hline & \multicolumn{2}{|l|}{ Cerramientos } & 1 \\
\hline & \multicolumn{2}{|l|}{ Cubiertas } & 2 \\
\hline \multicolumn{3}{|l|}{ Materialidad (9 puntos) } & 9 \\
\hline \multirow{18}{*}{ Estado de conservación (15 puntos) } & \multirow{6}{*}{ Estructura } & Muy bueno (5) & \multirow{6}{*}{1} \\
\hline & & Bueno (4) & \\
\hline & & Regular (3) & \\
\hline & & Malo (2) & \\
\hline & & Muy malo/ruina (1) & \\
\hline & & Ruina económica (o) & \\
\hline & \multirow{6}{*}{ Cerramientos } & Muy bueno (5) & \multirow{6}{*}{4} \\
\hline & & Bueno (4) & \\
\hline & & Regular (3) & \\
\hline & & Malo (2) & \\
\hline & & Muy malo/ruina (1) & \\
\hline & & Ruina económica (o) & \\
\hline & \multirow{6}{*}{ Cubiertas } & Muy bueno (5) & \multirow{6}{*}{3} \\
\hline & & Bueno (4) & \\
\hline & & Regular (3) & \\
\hline & & Malo (2) & \\
\hline & & Muy malo/ruina (1) & \\
\hline & & Ruina económica (o) & \\
\hline Medio Ambiente $( \pm 7)$ & & & o \\
\hline \multirow{2}{*}{ Valor de conjunto (10) } & \multicolumn{2}{|l|}{ Conjunto } & 5 \\
\hline & Paisaje industria & & o \\
\hline H & Maquinaria intac & & o \\
\hline Valor tecnologico (10) & Proceso producti & & o \\
\hline Apariencia original (5) & & & 5 \\
\hline Recurso revitalizador (5) & & & 5 \\
\hline Valoración total & & & $74 / 120$ \\
\hline
\end{tabular}


Tabla 2. Niveles de relevancia para un bien de Patrimonio Industrial.

\begin{tabular}{|c|l|l|}
\hline Niveles & \multicolumn{1}{|c|}{ Valoración total } & \multicolumn{1}{c|}{ Clasificación } \\
\hline 0 & $<30$ puntos & Bien industrial sin relevancia \\
\hline 1 & $31-60$ puntos & Valor general como Patrimonio Industrial \\
\hline 2 & $61-90$ puntos & Bien de relevancia industrial \\
\hline 3 & $91-120$ puntos & Bien de interés Histórico Industrial \\
\hline
\end{tabular}

Otro de los puntos de mayor importancia del trabajo, y en relación con la generación de documentación para el estudio sobre Patrimonio Industrial, es la generación de planos de la cúpula, hasta ahora escasos e inexactos, que se expondrá en el siguiente apartado.

\section{METODOLOGÍA EMPLEADA: MODELADO BIM}

Una parte de la documentación generada en esta investigación para la valoración de la cúpula de la ETSII ha sido obtenida empleando herramientas basadas en metodología BIM (Building Information Modelling o Modelado de Información de la Edificación), que han permitido trabajar con un amplio abanico de características del edificio, y elaborar un modelo digital muy detallado de dicha construcción, incorporando en el mismo los rasgos particulares con los que cuenta. Estos factores han sido desde las propiedades geométricas o físicas principales de los materiales empleados, como densidades o coeficientes térmicos, hasta información relativa a las instalaciones existentes, lo que incluye tanto su trazado y equipos (válvulas del sistema antiincendios, cuadros de luz, conductos de ventilación...), como sus características técnicas.
El Comité Nacional de Norma BIM de Estados Unidos define el concepto BIM como: “...una representación digital de las características físicas y funcionales de un complejo. Un BIM es una fuente de conocimiento compartido sobre un conjunto que permite generar una base fiable para la toma de decisiones durante su ciclo de vida, definido éste como el tiempo entre la idea original de su construcción hasta su demolición". (17)

Este tipo de análisis se utiliza para planificar, diseñar, construir, operar y mantener, e incluso desmantelar infraestructuras tan diversas como: servicios eléctricos, sanitarios y aire acondicionado, pero también casas, carreteras, puentes, puertos, escuelas, oficinas, almacenes o fábricas.

En general, el trabajo empleando herramientas BIM comienza con un modelo completo del conjunto de la obra (o del problema sobre el que se necesita trabajar) mediante software y resulta interesante destacar el carácter colaborativo y multidisciplinar que permite posteriormente. A partir de este modelo principal, se favorece la participación de ingenieros, arquitectos, y técnicos expertos en instalaciones, que pueden

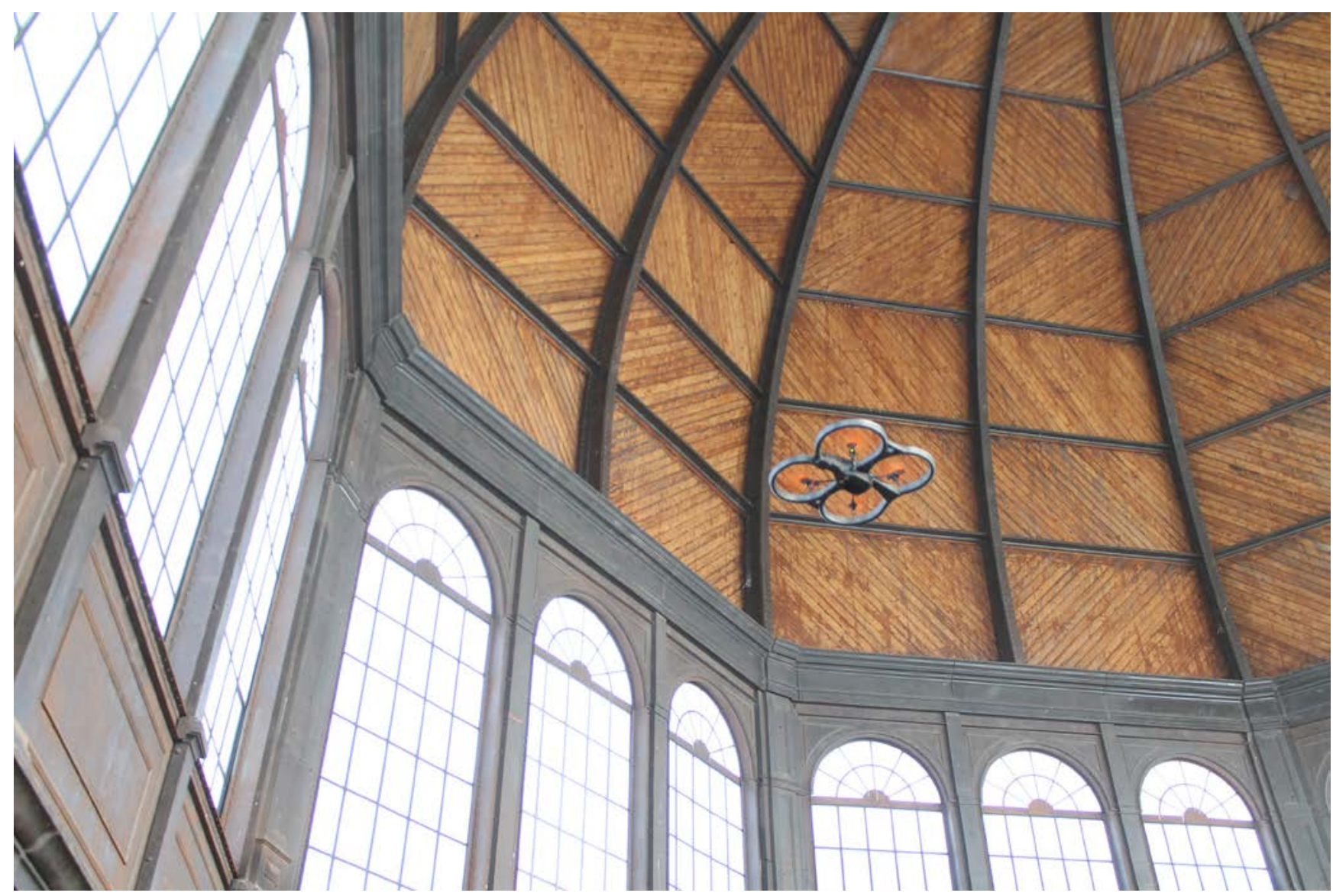

Figura 6. Quadrator empleado para la reconstrucción del interior de la cúpula. Fuente: Autores. 

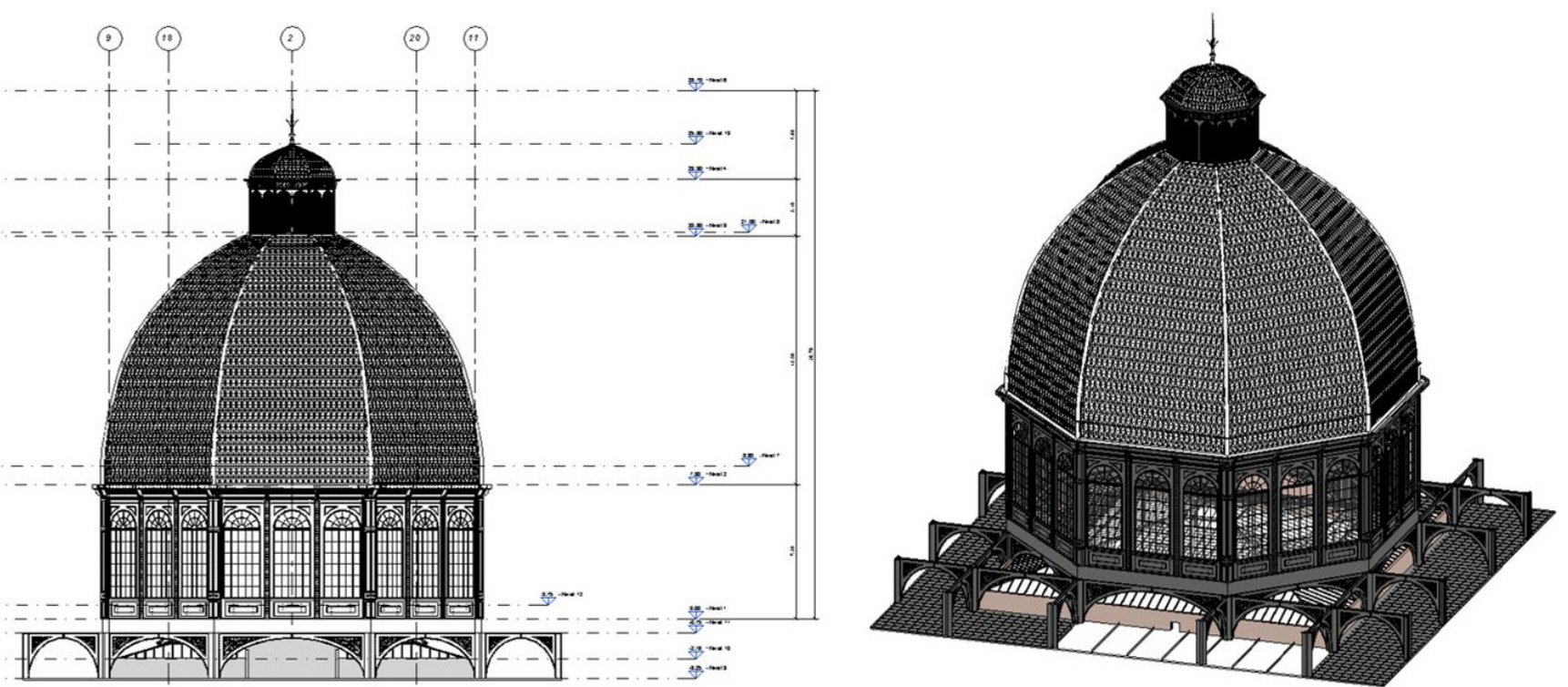

Figura 7. Vista en perspectiva y alzado de la cúpula en Revit.

consultar y editar el modelo original simultáneamente y de forma deslocalizada.

El software utilizado ha sido el paquete Autodesk: Revit versión 2014; ya que admite la construcción del modelo en tres dimensiones con un alto nivel de detalle y realismo y la recogida de una gran cantidad de información.

La primera acción llevada a cabo ha sido la búsqueda de todos los planos disponibles relativos a la cúpula. Los más recientes se corresponden con el proyecto de restauración del año 2007 (6), donde tras una tormenta las tejas de la cúpula se desprendieron debiendo ser sustituidas las tejas de zinc originales por otras nuevas. Esta documentación era inexacta, y ha sido imprescindible realizar visitas a la cúpula para tomar fotografías y medidas directamente con la intención de minimizar los posibles errores y generar unos planos fiables para el futuro.

Durante la primera visita se contó con la ayuda del Departamento de Automática, Ingeniería Eléctrica y Electrónica e Informática Industrial de la ETSII, que cedió un quadrotor para escanear el interior y grabar vídeos de zonas de difícil acceso, como por ejemplo la pasarela superior ubicada en el interior del cupulín, situada a casi 21 metros de altura inaccesible en los momentos actuales (Figura 6).

Tomando estos datos como referencia, se ha procedido a generar los planos en tres dimensiones en Revit con un alto nivel de detalle para después añadir los materiales y texturas para que el modelo fuese todo lo realista posible (Figura 7).

Los planos incluyen la cúpula y todos sus elementos, pero también el piso inferior, los arcos de soporte y el lucernario. Además, se han hecho archivos independientes que recogen el estado actual frente al previo a las remodelaciones de las que se tiene constancia.

El modelado 3D es otra de las tareas desarrolladas durante el trabajo pudiendo ser utilizado para llevar a cabo multitud de estudios futuros. Se trata por tanto de una herramienta básica que resultará muy útil en posibles análisis de eficiencia energética, remodelaciones o inclusión de nuevos refuerzos estructurales.

\section{CONCLUSIONES}

La evidente dependencia de la cúpula con el resto del edificio se ha tenido en cuenta a lo largo de todo el estudio, y se ha intentado aislar para que pueda ser valorada por separado. Sin embargo, en algunos casos ha sido inevitable acudir al conjunto de la edificación para tratar temas como la historia, las actividades desarrolladas o el valor arquitectónico. Este hecho podría indicar la necesidad de valorar todo el edificio en su conjunto, y no sólo la cúpula, en un proyecto posterior.

De acuerdo al sistema de puntuación planteado y después del análisis realizado siguiendo los criterios establecidos previamente, la cúpula de la Escuela Técnica Superior de Ingenieros Industriales ha obtenido una valoración de 74 puntos sobre un total de 120 posibles.

Esto implica que la cúpula puede ser considerada como un bien de relevancia industrial.

Con todo esto, resulta evidente la importancia de la cúpula no sólo para el edificio de la Escuela y el Museo de Ciencias Naturales, sino también para su entorno. El estado actual de la estructura requiere una pronta intervención para evitar la grave pérdida que supondría su desaparición. También se tienen argumentos suficientes para demostrar que la inversión económica necesaria para llevar a cabo esta actuación podría obtenerse a partir de la inclusión de la cúpula en el catálogo de bienes de Patrimonio Industrial.

\section{AGRADECIMIENTOS}

Este trabajo se encuadra dentro de las actividades del Aula de Formación "Gestión e Intervención sobre el Patrimonio de la Arquitectura y la Industria G+I_PAI” promovida por la Fundación ACS y la Universidad Politécnica de Madrid. Agradecemos a Raquel de la Heras las copias de los planos originales del edificio que nos proporcionó. 


\section{REFERENCIAS}

(1) Plan Nacional de Patrimonio Industrial, marzo 2011. http://ipce.mcu.es/pdfs/PN_PATRIMONIO_INDUSTRIAL.pdf

(2) La Ilustración Española y Americana, (1887) "Madrid - Exposición Nacional de Bellas Artes", año XXXI - número XIX, Madrid, 22 mayo.

(3) Dabrio Soldán, Miriam; R. Pujazón, Rocío; (2014) “Arquitectura del hierro y sistema Polonceau para un nuevo concepto de mercado”, Huelva Buenas Noticias. http://huelvabuenasnoticias.com/2014/o6/13/arquitectura-del-hierro-y-sistema-polonceau-para-un-nuevo-concepto-de-mercado/, 13 de junio.

(4) Museo Nacional de Ciencias Naturales. http://www.mncn.csic.es/Menu/Elmuseo/Eledificio/seccion=1178\&idioma=es_ ES.do

(5) Blanc Díaz, Manuel; (1963) “Memoria de Proyecto de reforma de la Escuela”, diciembre.

(6) II Congreso Internacional de Arquitectos y Técnicos de Monumentos Históricos "Carta Internacional para la Conservación y Restauración de Monumentos y Sitios” o "Carta de Venecia”, Venecia, 25 mayo 1964. Revisado en 1978.

(7) García del Monte, José $\mathrm{M}^{\mathrm{a}}$; Montiel, Ana (2007) “Proyecto de rehabilitación de la cúpula de la ETSIIM”. Obras de emergencia. QVE Arquitectos.

(8) Gámez Mejías, Linarejos, (2007) “Informe de Rehabilitación Cúpula ETSII”, 8 noviembre.

(9) Escuela Técnica Superior de Ingenieros Industriales de MADRID (2009) "Plan Director de la Escuela Técnica Superior de Ingenieros Industriales de Madrid. Restauración de la cúpula”, diciembre.

(10) G. Blázquez, Manuel (2008) "La cúpula más brillante de la Castellana”, Artículo en El País, 24 octubre.

(11) Convención sobre la Protección del Patrimonio Mundial Cultural y Natural de la UNESCO, París, 16 noviembre 1972.

(12) Comité de Monumentos y Sitios del Consejo de Europa (1975) "Carta Europea del Patrimonio Arquitectónico".

(13) $14^{\mathrm{a}}$ Asamblea General del ICOMOS "Principios para el análisis, conservación y restauración de las estructuras del Patrimonio Arquitectónico”, Victoria Falls, octubre 2003.

(14) Cortes Generales (1985). Ley 16/1985 de Patrimonio Histórico Español, 25 junio 1985. Boletín Oficial del Estado de 29 de junio..

(15) Casanelles i Rahóla, Eusebi (2007) "Nuevo concepto de Patrimonio Industrial, evolución de su valoración, significado y rentabilidad en el contexto internacional”, "Bienes Culturales”. Instituto del Patrimonio Histórico Español”, nº 7.

(16) Diana Sánchez Mustieles (2012) "Metodología para la recuperación y puesta en valor del patrimonio industrial arquitectónico. Antiguas fábricas del Grao de Valencia”, Tesis Doctoral. Universidad Politécnica de Valencia.

(17) National BIM Standard (2014) "Frequently Asked Questions About the National BIM Standard - United States", 17 October. 